\title{
A Novel Mobile Communication System using Pulse Position based Chirp Spread Spectrum Modulation
}

\author{
Ádám Knapp and László Pap
}

\begin{abstract}
The paper presents a new mobile communication system based on Chirp Spread Spectrum (CSS) transmission. The downlink modulation scheme is extended with Pulse Position Modulation (PPM) to carry data for multiple mobile terminals simultaneously. The described novel mechanism ensures reliable and robust communication between the parties, especially for terminals moving with high speeds or at long range. Furthermore, the proposed system take care of the uplink communication as well, where Closed-Loop Power Control (CLPC) is applied to handle the near-far problem and improve the performance of the system. Based on the attributes of the proposed system the application area covers sensor networks, IoT applications and Industry 4.0 as general field of LPWAN, however, mobility of terminals also considered. Analytical investigations for downlink communication are described focusing on the instantaneous symbol-error rate and average SER in Rayleigh fading channel. The results show that the proposed Pulse Position based Chirp Spread Spectrum technique for Multiple Access (shortly PP-CSSMA) allows higher data rates that is used for the multiple access feature. In addition, numerical results are presented as well, and they point out the benefits of the applied CLPC mechanism. Finally, considerations regarding to the implementation of the proposed communication system are described.
\end{abstract}

Index Terms-LPWAN, Chirp Spread Spectrum, Pulse Position Modulation, Multiple Access, Symbol-Error Rate, Rayleigh fading.

\section{INTRODUCTION}

As technology getting bigger part of our everyday's life, new application areas emerge such as Internet of Things (IoT), Industry 4.0 and every kind of "smart" things like smart metering, smart home, smart city, smart agriculture etc. [1][2]. These bring new requirements and challenges to the field of telecommunication mostly due to the different nature of communicating parties. Machine-to-Machine (M2M) or Machine Type Communication (MTC) is one of the key enablers of highly automated procedures for the mentioned applications. In this paper the concept of a novel mobile communication system is presented. While usual Low-Power Wide Area Networks (LPWAN) technologies handle fixed devices that are often operated from batteries, mobile terminals

Manuscript received July 16, 2018; revised August 31, 2018. Date of publication September 17, 2018. Prof. Nikola Rožić has been coordinating the review of this manuscript and approved it for publication.

Authors are with the Department of Networked Systems and Services, Budapest University of Technology and Economics, 1117, Budapest, Magyar tudósok krt. 2, Hungary.

E-mails: \{knapp,pap\} @ hit.bme.hu

Digital Object Identifier (DOI): 10.24138/jcomss.v14i3.582 are not considered. The proposed system is similar to LPWAN, however, it addresses different use cases where terminals are able to move, for example, factory logistic vehicles, even with high speeds, like unmanned aerial vehicles (UAV). Such use cases can be found for fifth generation mobile communication networks $(5 \mathrm{G})$ as well. One of the key domains of $5 \mathrm{G}$ is M2M communication, however, the complexity, the energy efficiency and the price of receivers makes $5 \mathrm{G}$ an uncompetitive technology on the field of LPWAN [3]-[5]. The proposed communication system applies Pulse Position based Chirp Spread Spectrum technique for Multiple Access (PP-CSSMA) in the downlink communication and PP-CSS with measurement based feedback power control for uplink. The used Closed-Loop Power Control (CLPC) mechanism is a channel equalization technique that is performed at the transmitter side, in this case by the mobile terminal, based on the measured and feedback distortion of the radio channel. The elegant and sophisticated combination of PP-CSS-MA and CLPC techniques give the novelty of the proposed mobile communication system. Therefore, the following paragraphs briefly summarize the history and current status of the mentioned mechanisms. Spread Spectrum (SS) and CLPC techniques are well-known from decades ago. SS communication is widely spread, standardized in many ways, and different forms are applied in vast of communication devices. On the one hand, the communication are based on Direct-Sequence Spread Spectrum (DSSS) technique in IEEE 802.11b standards, as the original Wi-Fi system; in IEEE 802.15.4 standard, which is the physical layer for ZigBee; in Code Division Multiple Access (CDMA) channel access method and in different Global Navigation Satellite Systems (GNSS) like GPS, GLONASS and Galileo. On the other hand, Adaptive Frequency-hopping spread spectrum (AFH) is applied in Bluetooth, which is a variant of Frequency-Hopping Spread Spectrum (FHSS) technique. Finally, Chirp Spread Spectrum is important part of IEEE 802.15.4a standard as a supported physical layer in LowRate Wireless Personal Area Networks (LR-WPAN) [6][7] and of LoRaWAN (Long Range Wide Area Network), which is an emerging communications technology for LPWAN as well [8][9]. The reasons can be found among the advantages of CSS, which include high robustness against to channel noise, interference and jamming. Furthermore, the resistance capability against the Doppler effect makes CSS a very good candidate in such mobile communication environment, where 
support for terminals moving with high speeds and at long ranges is necessary.

The importance of Closed-Loop Power Control has been shown by the traditional cellular mobile communication networks. It handles the so-called near-far problem, which is a quite common issue in mobile networks. CLPC is part of the operation in $2 \mathrm{G} \mathrm{GSM}$ as well, however, its significance improved in 3G CDMA-based cellular systems, which utilised CLPC to mitigate in-system interference.

The rest of the paper is organised as follows. Section II presents the related works, then Section III describes the system model and details the variant of the PP-CSS-MA technique and the applied CLPC method. Performance analysis and numerical results are presented in Section IV. Further discussion regarding practical aspects, benefits and drawbacks of the proposed system is described in Section V. Finally, Section VI concludes the paper.

\section{RELATED WORKS}

This section describes the most significant works and solutions related to the proposed PP-CSS-MA and CLPC techniques.

\section{A. Chirp Spread Spectrum}

The first usage of chirp signals dates back to the World War II, where it was applied in radar technology for its unique properties of pulse compression and precise ranging [10]. Since then numerous papers were published about extending chirp modulation with multiple access. In 1974, multi-access CSS was introduced by Cook [11]. He analyzed the utility of pairs of linear chirps with different chirp rates as spread spectrum signals by assigning them to several users. This enabled multiple access within a common frequency band. Then, this approach was extended by El-Khamy et al. in 1996 [12]. The authors proposed binary multi-user communication scheme by selecting the chirp signals in a way, where they all have the same power as well as the same bandwidth. Similar trend is visible in later papers, which are about extending chirp modulation with multiple access. All of them [13]-[16] focus on making the chirp signals distinguishable based on changing their different attributes, e.g. the slope, initial phase etc. Authors of [14] and [15] also take into consideration the orthogonality to enhance the performance of the proposed CSS based communication systems. Contrarily, the approach of the proposed PP-CSS-MA technique resembles the work of ElKhamy et al. from 1999 [17], in which the authors propose a multi-user communication system extended with frequency hopping. The benefit of this hybrid spread spectrum technique is that it improves the communication system performance especially in multipath fading dispersive channels.

Furthermore, LoraWAN has to be mentioned as well, since it is a similar, emerging LPWAN technology. It mostly focuses on low-power, usually battery operated sensors to be the communication enabler for such applications as smart metering, smart cities, etc. Thy physical layer of LoRa is based on Chirp Spread Spectrum using flexible configurability of the modulation parameters [18]. Table I contains LoRa CSS
Table I

LORA CSS MODULATION PARAMETERS

\begin{tabular}{|c|c|}
\hline Parameter & Values \\
\hline Spreading Factor (SF) & $6-12$ \\
\hline Code Rate (CR) & $1-4$ \\
\hline Bandwidth (BW) $[\mathrm{kHz}]$ & $10.4,15.6,20.8,31.25,41.7,62.5,125,250,500$ \\
\hline
\end{tabular}

modulation parameters, and the nominal bit rate is expressed by Eq. 1.

$$
R_{b}=S F \times 1000 \times \frac{\left[\frac{4}{4+C R}\right]}{\left[\frac{2 S F}{B W}\right]}
$$

It is visible that LoRaWAN is complex communication system, which aims to provide service for hundreds of nodes. Therefore, it applies unslotted ALOHA as channel access method. The possible limitations of that are discussed in [19].

\section{B. Closed-Loop Power Control}

Closed-loop power control solves the near-far problem primarily, while channel equalization compensates the influence of random fluctuation (fading) appearing in the radio channel. Traditionally, the prior problem is tackled by high delay closed-loop power control system, contrarily, the solution of the latter one requires diversity techniques [20]. The significance of CLPC is shown in 3G CDMA based mobile communication technologies due to the need for coordinating in-system interference. Furthermore, it is integral part of $4 \mathrm{G}$ LTE/LTE-A systems especially their MIMO related operations [21]. It has central role in other kind of mobile communication systems, namely in cognitive radio systems [22], [23]. Lots of research have focused on the performance of separated closed-loop power control system and diversity techniques to solve the near-far problem and the channel equalization in presence of white Gaussian noise and some special types of intentional interference. The most relevant to the present paper are those that investigate the error rate of the closedloop power control scheme for the multi-rate services in the third and forth generation wideband systems. The authors of [24] proposed an algorithm that computes the solution of the power control problem with closed-loop effects. Furthermore, analytical and simulation results are presented that show the convergence of the algorithm under the same conditions as that given in the earlier results. In [25] smart step closed-loop power control (SSPC) algorithm introduced in a DS-CDMA receiver assuming frequency-selective Rayleigh fading. The proposed receiver comprises three phases. In the first phase the desired signal of users in an arbitrary path is passed and the inter-path interference (IPI) is reduced. Also in this phase, the multiple access interference (MAI) from other users is reduced. Thus, the matched filter can be used for the MAI and IPI reduction in the second phase. Then, the output signals from the matched filters are combined according to the conventional maximal ratio combining (MRC) principle in the third phase. Finally, these are fed into the decision circuit of the desired user. 


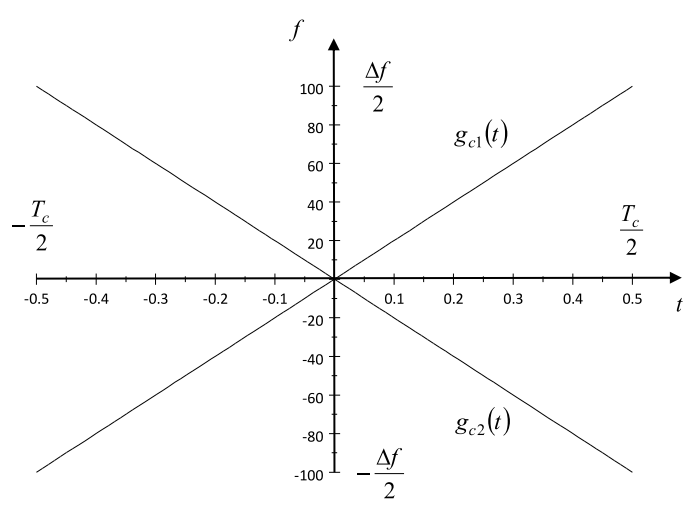

Figure 1. Instantaneous frequency of the chirp signal in function of time, when $T_{c}=1, \Delta f=200$

\section{SySTEM MODEL}

This section introduces the basic operation of mobile communication system. The downlink is based on the PP-CSS-MA technique, while the system applies PP-CSS on the uplink with measurement based CLPC method. The mathematical representation of these techniques are presented in the subsections.

Assume a communication system, which provides wireless connectivity between a base station (BS) and mobile terminals (MT). The available bandwidth of the system is fully utilised by Chirp Spread Spectrum transmission, where the signals are modulated by chirp pulses, i.e. frequency varying sinusoidal pulses. To simplify the RF circuit design time-division duplexity is applied in the system to separate the downlink and uplink communication. Since the focus is on the improvement of CSS using pulse-position for multiple access on the downlink and on the measurement based feedback channel equalization on the uplink, every aspect of the communication system's operation is not detailed in this paper.

\section{A. Downlink: PP-CSS-MA}

This subsection presents two, slightly different versions of Pulse-Position based Chirp Spread Spectrum for Multiple Access.

The following expression gives the low-pass equivalent complex-valued representations of the elementary chirp signals:

$$
\text { for } 0: \quad \begin{aligned}
g_{c 1}(t) & =A \exp \left(j 2 \pi \frac{\Delta f}{2 T_{c}} t^{2}\right) \\
\text { for } 1: \quad g_{c 2}(t) & =A \exp \left(-j 2 \pi \frac{\Delta f}{2 T_{c}} t^{2}\right), \\
t & \in\left[-\frac{T_{c}}{2}, \frac{T_{c}}{2}\right)
\end{aligned}
$$

where $A$ is the amplitude of the elementary signals, $\Delta f$ is the chirp modulated signal's frequency spreading domain and $T_{c}$ is the frequency varying sinusoid signal's symbol-time. The instantaneous frequency changing of the signal is presented on Fig. 1. The units of the time ( $x$-axis) and frequency domain ( $y$-axis) are $10^{-5}$ second and $10^{5} \mathrm{~Hz}$, respectively.

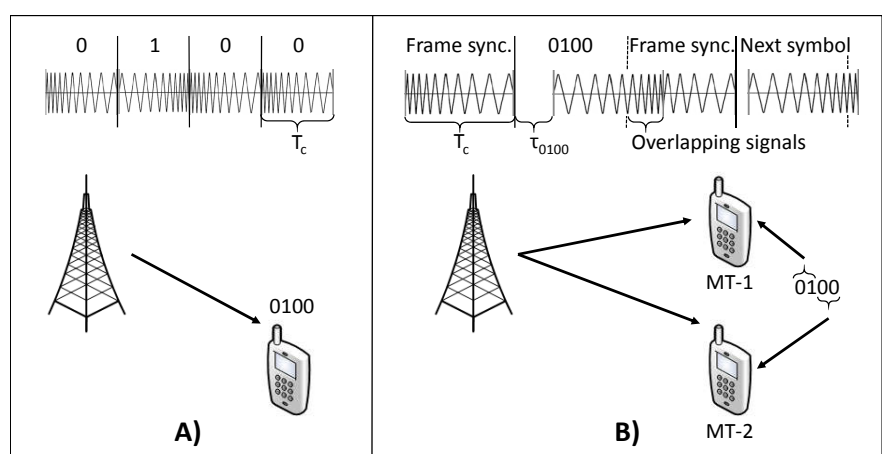

Figure 2. Model of the original CSS (A) and the extended PP-CSS-MA (B) based communication systems (variant A)

1) PP-CSS-MA variant A: To extend the downlink chirp spread spectrum communication with pulse position modulation, framing is necessary in order to the terminals be able to distinguish the transmitted bits from the BS. In the present paper, it is supposed that the elementary down-chirp signal is applied to indicate the starting of the frame, however, it is also possible to use the up-chirp signal for this purpose.

Let $g_{c 0}(t)$ the elementary signal for frame synchronization and $g_{c 1}(t), g_{c 2}(t), \ldots, g_{c i}(t), \ldots, g_{c 2^{M}}(t)$ the elementary signals for data symbols in the non-binary pulse position based chirp modulation system. The next expression describes the low-pass equivalent complex-valued representations of these signals:

$$
\begin{aligned}
g_{c 0}(t)= & A \exp \left(-j 2 \pi \frac{\Delta f}{2 T_{c}}\left(t-\tau_{0}\right)^{2}\right), \\
& \left(t-\tau_{0}\right) \in\left[-\frac{T_{c}}{2}, \frac{T_{c}}{2}\right) \\
g_{c i}(t)= & A \exp \left(j 2 \pi \frac{\Delta f}{2 T_{c}}\left(t-\tau_{i}\right)^{2}\right), \\
& \left(t-\tau_{i}\right) \in\left[-\frac{T_{c}}{2}, \frac{T_{c}}{2}\right)
\end{aligned}
$$

where $\tau_{i}$ is the delay related to the time position of each symbol, $i=1, \ldots, 2^{M}$ is the symbol number and $2^{M}+1$ is the overall number of the available symbols in the system. $M$ is referred later as the data rate parameter. Other notations are identical to ones in Eq. 2.

The equation describes that the useful information carrying symbols are generated using proper time-shifted up-chirp signals. Due to the applied chirp modulation, this kind of time-shifted signals have the important attribute that in a time slot (with given delay) there is only one elementary signal available that has the appropriate even and odd auto- and crosscorrelation according to the other symbols. With other words, CSS allows to use quasi-orthogonal signals with different, $2^{M}$ possible delays to transmit $2^{M}$ possible data symbols. These, well-distinguishable symbols can provide the multiple access feature of the system.

The present variant, which denoted by $\mathrm{A}$, is about that $2^{M}$ different data symbol are mapped to $M$-length binary words similarly to the gray mapping in case of PSK modulations. The mapping itself is not considered in the paper. The principle is simply that the $M$-bit transmitted information using the 


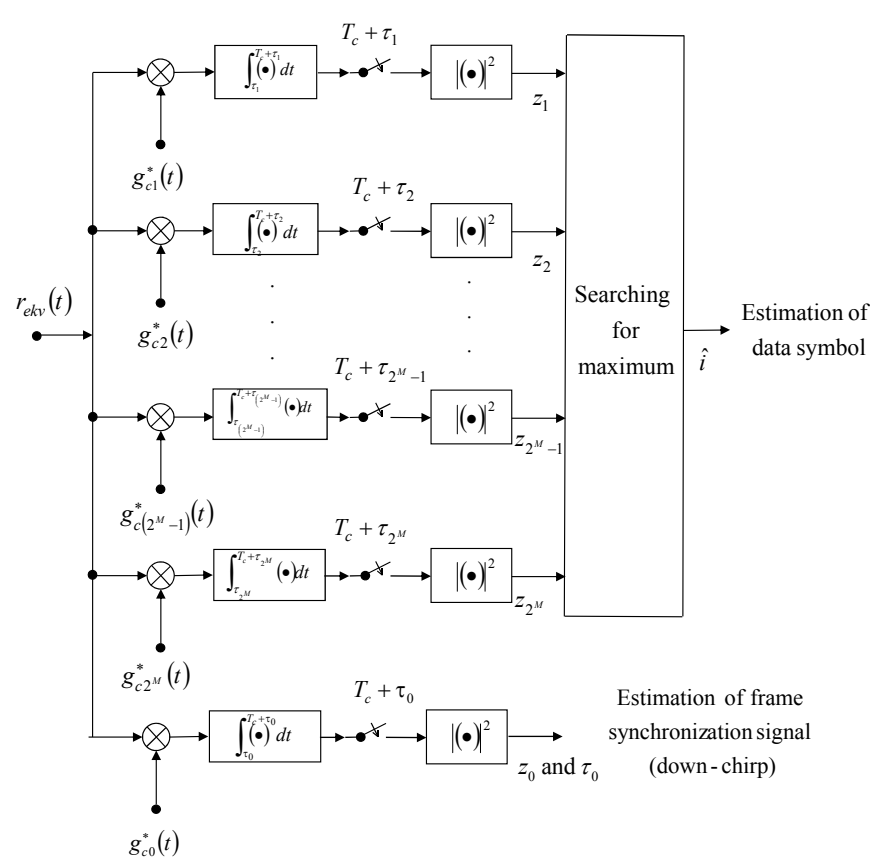

Figure 3. The structure of the proposed non-coherent PP-CSS-MA variant A receiver using non-binary chirp modulated signals

$2^{M}$ different symbols are separated and assigned based on a predefined pattern to at most $M$ mobile terminals. One possible pattern is when a bit or a part of the transmitted $M$ length binary word is associated to a mobile terminal based on its sequence number or identifier. The operation of the traditional CSS based method and the proposed PP-CSS-MA (variant A) technique are illustrated in Fig. 2.

For better understanding, let's look the following example. Assume that the system configured with the data rate parameter $M=4$ and there are the same number of mobile terminals. If the BS sends the elementary symbol related to 0100, all the MTs will receive and demodulate it. Then the first, third and fourth terminal will get the 0 and the second the 1 binary information. Note, it is also possible to assign the 4-length binary word as the first MT gets the first 01 part and the second terminal the second 00 part of the transmitted word, when there are only two terminals in the system. The latter case is depicted in Fig. 2 B. Of course, other allocation patterns can be applied for the separation. Just a reminder, to transmit $M=4$ length binary words by the base station, $2^{M}=16$ different, time-shifted up-chirp signals are necessary (see Eq. 3).

The structure of the proposed non-coherent PP-CSS-MA variant A receiver using non-binary chirp modulated signals is illustrated in Fig. 3. Further details about PP-CSS-MA variant $\mathrm{A}$ are available in [26].

2) PP-CSS-MA variant B: By extensively investigating the possibilities, a slightly different multiple access scheme is presented as well. Instead of mapping the time-shifted signals to binary words, which then are bitwise or partially assigned to the mobile terminals, the idea is to map the time-shifts directly to MTs. One $\tau$ delay is required to be associated to a terminal, the up- and down-chirps related the given delay as pairs carry the binary information for the MT. In other words, in this case

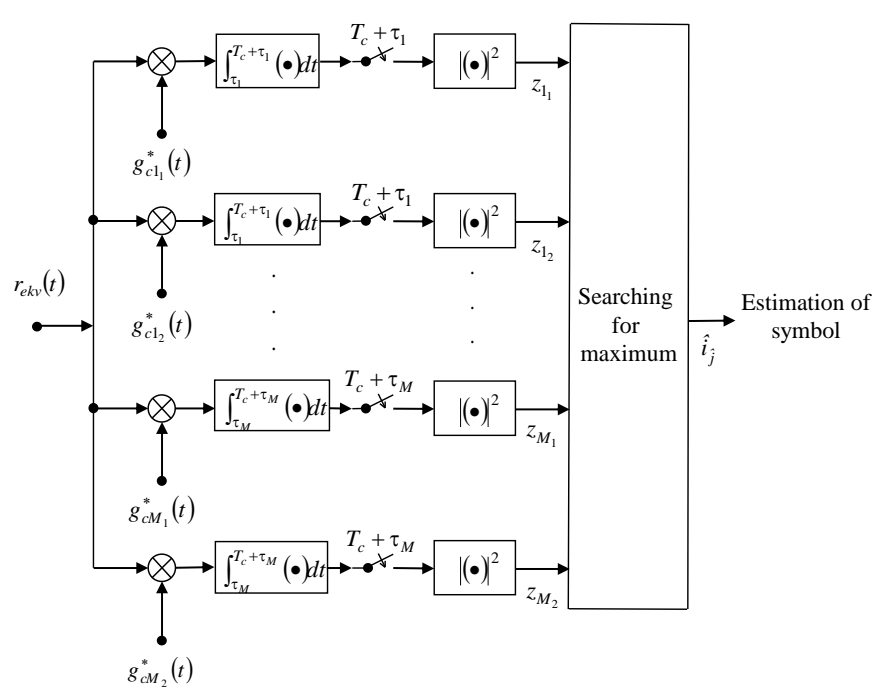

Figure 4. The structure of the proposed non-coherent PP-CSS-MA variant B receiver using non-binary chirp modulated signals

the base station is able to send $M$ number of different timeshifted up- and down-chirp signals, however, only one mobile terminal will receive one bit information depending on the $\tau$-shift. The exact value of the binary information is decided based on the direction of the given, transmitted signal. Framing is not necessary in this variant.

$$
\begin{aligned}
& g_{c i_{1}}(t)= A \exp \left(-j 2 \pi \frac{\Delta f}{2 T_{c}}\left(t-\tau_{i}\right)^{2}\right), \\
&\left(t-\tau_{0}\right) \in\left[-\frac{T_{c}}{2}, \frac{T_{c}}{2}\right) \\
& g_{c i_{2}}(t)=A \exp \left(j 2 \pi \frac{\Delta f}{2 T_{c}}\left(t-\tau_{i}\right)^{2}\right), \\
&\left(t-\tau_{i}\right) \in\left[-\frac{T_{c}}{2}, \frac{T_{c}}{2}\right)
\end{aligned}
$$

where $i=1, \ldots, M$ is the symbol number and $2 M$ is the overall number of the available symbols in variant B. Other notations are the same as in Eq. 3.

Let's look another example. For the sake of simplicity, now assume two MTs in the system and the preconfigured data rate parameter is $M=2$. In this case, there are $M=2$ different, time-shifted up- and down-chirp signals to carry useful information. Let's use the following mapping: $\tau_{1}$ delay (i. e. $g_{c 1_{1}}$ and $g_{c 1_{2}}$ elementary signals) is assigned to the first terminal and $\tau_{2}$ delay (i.e. $g_{c 2_{1}}$ and $g_{c 2_{2}}$ elementary signals) is associated to the second terminal. According to this mapping if the base station sends the $\tau_{1}$-shifted up-chirp $\left(g_{c 1_{1}}\right)$, then the first mobile terminal will receive the binary 0 . In other case, when the base station sends the $\tau_{2}$-shifted down-chirp signal $\left(g_{c 2_{2}}\right)$, then the second MT will receive the binary 1 .

Fig. 4 presents the structure of the proposed non-coherent PP-CSS-MA variant B receiver using non-binary chirp modulated signals. As mentioned earlier in this case framing is not necessary that reflects the receiver structure as well.

In the following the correlation properties of elementary signals of PP-CSS-MA variant B technique are detailed to 


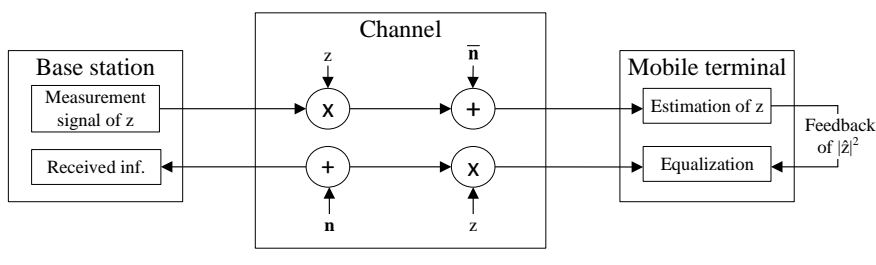

Figure 5. System model of the proposed measurement based feedback power control mechanism

justify the quasi-orthogonality between them.

\section{B. Uplink: PP-CSS using CLPC}

This subsection describes the uplink communication of the proposed system including the proposed closed-loop power control mechanism.

On the uplink the same signals are used, but in this case the common channel is shared in time between the mobile terminals. Since the symbol synchronization is defined by the base station, each MT transmits in its own, dedicated time slot using pulse position based chirp spread spectrum modulation. The $i-t h$ mobile terminal can send its binary information using the assigned elementary signals. According to PP-CSSMA variant $A$, these signals can be the up-chirps starting with different time delays (Eq. 3) or they can be both up- and downchirps with identical time-shift in case of variant B (Eq. 4). Hence, the overall throughput of MTs is $M$.

The signals of different mobile terminals can overlap at the receiver of the $\mathrm{BS}$, however, the quasi-orthogonal property of the signals allows their efficient separation. It is important that the incoming signals are not fully orthogonal, thus they interfere with each other that can be amplified by the near-far problem. This means that the MTs usually are in different distances from the base station, therefore the wireless signals arrive with different amplitudes (and with different time delays) into the antenna of the BS during the uplink communication. Hence, a closer MT's signal can suppress the farer one's. To handle this issue and control the amplitude of the received signals, closed-loop power control mechanism is applied in the transmitter of MTs. Nevertheless, the system is insensitive to the estimation error of the amplitude's level due to low odd and even cross-correlation between the signals (see next section), but assuming a noisy and fading channel, where the estimation is not errorless, the bit-error rate of the communicating system is strongly influenced. Therefore, the measurement based feedback power control method is applied on the uplink to ease the proper reception in the base station. This CLPC mechanism is detailed in [27], which contains its performance analysis using the non-coherent binary transmission as well. In addition, the investigations cover Rayleigh, Rice and Nakagami fading types, too. In the current paper its slightly changed version is used, but the presented results are still applicable in this case. The system model of the proposed CLPC mechanism is illustrated in Fig. 5.

The low-pass equivalent complex-valued representation of the binary modulated signal $\mathbf{r}^{*}$ without channel equalization at the base station's front end during a slot is given as follows:

$$
\mathbf{r}^{*}=\sqrt{E_{s}} \mathbf{g}^{(b)} z+\mathbf{n}, \quad t \in(0, T],
$$

where $E_{s}$ is the average symbol energy without fading and power control, $T$ is the symbol time, $b \in[0,1]$ is the binary symbol, $\left\{\mathbf{g}^{(b)},\left\|\mathbf{g}^{(b)}\right\|^{2}=1\right\}$ are the low-pass equivalent complex-valued representation of the elementary signals in non-coherent case $\left\langle\mathbf{g}^{(0)}, \mathbf{g}^{(1)}\right\rangle=0, z$ is the complex fading channel parameter and $\mathbf{n}$ is the low-pass equivalent complexvalued representation of the additive white Gaussian noise with $\mathbb{E}\left[\mathbf{n n}^{*}\right]=N_{0} \mathbf{I}$, where $\mathbf{I}$ is the unity matrix. $z$ is a random variable that represents the instantaneous amplitude of the received binary signal $\left(\mathbb{E}\left[|z|^{2}\right]=1\right)$. The distribution of $z$ depends on the fading scenario, in the present case non-selective slow Rayleigh fading model is considered. It is supposed that the fading channel parameter is estimated in the transmitter of the mobile terminals by measuring an independent pilot channel, which has the same fading parameter as the communication channel. In this case the pilot and communication channels are the same ensuring that the fading is identical, however, the additive noise is assumed to be independent during the downlink and uplink communication. Furthermore, in case of PP-CSS-MA variant A the down-chirp signal used for frame synchronization is a good candidate to be the pilot signal for the proposed measurement based feedback power control mechanism.

\section{Correlation Properties of Elementary Signals IN PP-CSS TECHNIQUE}

In this subsection the mathematical investigation is introduced at the receiver side of the proposed PP-CSS communication system.

To simplify the analysis, the correlation of $g_{c 1_{1}}$ (i.e. upchirp signal related to $\tau_{1}$ delay) and $g_{c 1_{2}}$ (i.e. down-chirp signal related to the identical delay) elementary signals will be analyzed, because the other elementary signals can be derived from these by time shifts.

To detect the transmitted symbols in the receiver, the peak positions of the chirp signals related to the correlator's output $z_{1_{1}}$ and $z_{1_{2}}$ has to be detected. Thus, the behavior of the pulse is relevant, when the signal is delayed according to the symbol synchrony. This behavior is described by the formula below:

$$
\begin{aligned}
z_{1_{1}}(\tau)=\mid & \int_{-\frac{T_{c}}{2}}^{\frac{T_{c}}{2}-\tau} A e^{j \theta} g_{c 1}(t+\tau) g_{c 1}^{*}(t) d t+ \\
& \left.\int_{\frac{T_{c}}{2}-\tau}^{\frac{T_{c}}{2}} A e^{j \theta} g_{c 1}(t+\tau) g_{c 1}^{*}(t) d t\right|^{2}, \\
z_{1_{2}}(\tau)=\mid & \int_{-\frac{T_{c}}{2}}^{\frac{T_{c}}{2}-\tau} A e^{j \theta} g_{c 1}(t+\tau) g_{c 1}^{*}(t) d t+ \\
& \left.\int_{\frac{T_{c}}{2}-\tau}^{\frac{T_{c}}{2}} A e^{j \theta} g_{c 1}(t+\tau) g_{c 1}^{*}(t) d t\right|^{2},
\end{aligned}
$$

where $\tau$ is the delay, and where the output of the non-coherent detector related to the up-chirp signal is analyzed twice, when the incoming signal contains two, identical up-chirps $\left(z_{1_{1}(\tau)}\right)$ 


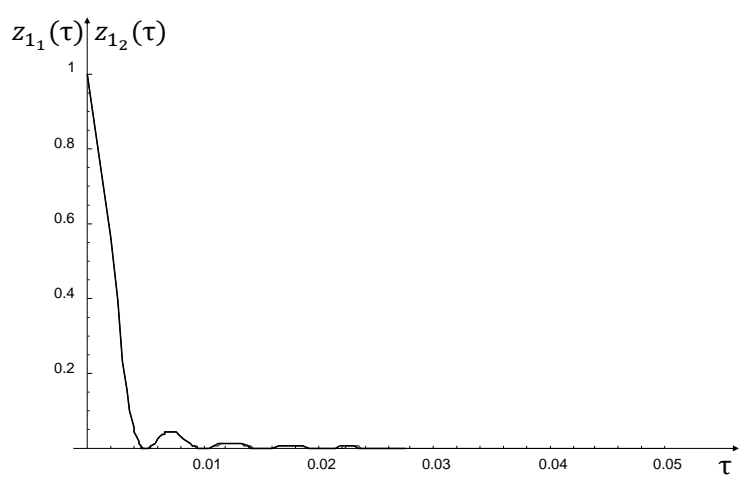

Figure 6. The output pulses $\left(z_{1_{1}}\right.$ and $\left.z_{1_{2}}\right)$ of the correlator related to the up-chirp signal in the non-coherent binary chirp receiver

and when it includes one up-chirp and one down-chirp $\left(z_{1_{2}(\tau)}\right)$ in $\left[-T_{c} / 2, T_{c} / 2\right)$ domain. The solution of these equation is given in Fig. 6, when $T_{c}=1, \Delta f=200, A=1$ and the symbol energy is $E_{s}=1 / 2$. It is clearly visible, that the correlation peak can be detected very accurately allowing to determine the symbol synchrony of the chirp signal very accurately as well.

Besides, it is necessary to investigate the output of the other detector described by the next expression:

$$
\begin{aligned}
z_{2_{1}}(\tau)=\mid & \int_{-\frac{T_{c}}{2}}^{\frac{T_{c}}{2}-\tau} A e^{j \theta} g_{c 1}(t+\tau) g_{c 2}^{*}(t) d t+ \\
& \left.\int_{\frac{T_{c}}{2}-\tau}^{\frac{T_{c}}{2}} A e^{j \theta} g_{c 1_{1}}(t+\tau) g_{c 2_{1}}^{*}(t) d t\right|^{2}, \\
z_{2_{2}}(\tau)=\mid & \mid \int_{-\frac{T_{c}}{2}}^{\frac{T_{c}}{2}-\tau} A e^{j \theta} g_{c 1}(t+\tau) g_{c 2}^{*}(t) d t+ \\
& \left.\int_{\frac{T_{c}}{2}-\tau}^{\frac{T_{c}}{2}} A e^{j \theta} g_{c 2}(t+\tau) g_{c 2}^{*}(t) d t\right|^{2},
\end{aligned}
$$

In this case the output of the non-coherent detector related to the down-chirp signal is analyzed twice, when the incoming signal contains two, identical identical up-chirps $\left(z_{2_{1}(\tau)}\right)$ and when it includes one up-chirp and one down-chirp $\left(z_{2_{2}(\tau)}\right)$ in $\left[-T_{c} / 2, T_{c} / 2\right)$ domain. The results are depicted in Fig. 7 using the same values of the parameters.

Comparing Fig. 6 and Fig. 7, it is visible that the different chirp signals are almost orthogonal close to $\tau=0$, therefore only the proper correlator produces useful output pulse during the sampling, while the output of the other correlator is almost zero.

As mentioned before, the base station is able to select from $2 M$ different elementary signals to transmit during a $T_{c}$ long slot. However, the BS radiates only one signal at a time, i. e. the number of transmitted information carrying bits is $1+\log _{2} M$ in a slot.

In this case, the structure of optimal non-coherent receiver for CSS is essentially not differs from the general noncoherent receiver using orthogonal elementary signals. The only difference is that the integration required for decision have to be performed based on several $T_{c}$ long domains

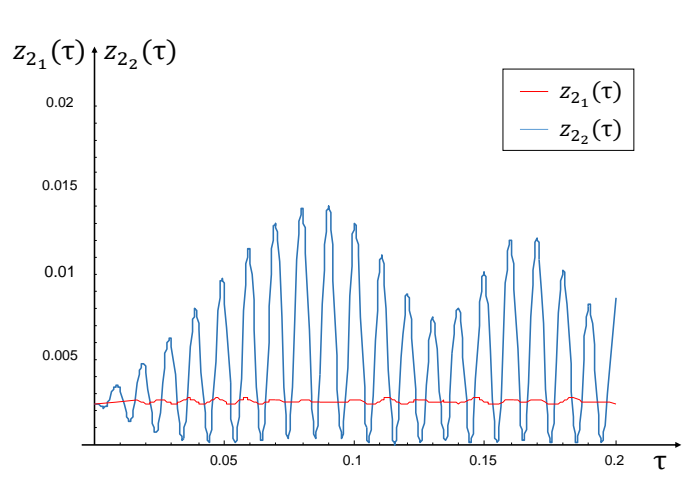

Figure 7. The output pulses $\left(z_{2_{1}}\right.$ and $\left.z_{2}\right)$ of the correlator related to the down-chirp signal in the non-coherent binary chirp receiver

starting in different time instants. In addition, in non-binary case the index of the maximum of $2 M$ decision signals $\left(z_{i_{1}}\right.$ and $z_{i_{2}}$ ) have to be determined. This non-coherent receiver is illustrated in Fig. 3, and its operation is represented by the following formulae:

$$
\begin{aligned}
z_{i_{k}}= & \left|\int_{-\frac{T_{c}}{2}+\tau_{i}}^{\frac{T_{c}}{2}+\tau_{i}} r_{e k v}(t) g_{c i_{k}}^{*}(t) d t\right|^{2}=\mid \int_{-\frac{T_{c}}{2}+\tau_{i}}^{\frac{T_{c}}{2}+\tau_{i}} r_{e k v}(t) \\
& \left.A \exp \left((-1)^{k} 2 j \pi \frac{\Delta f}{2 T_{c}}\left(t-\tau_{i}\right)^{2}\right) d t\right|^{2}
\end{aligned}
$$

where $i=1, \ldots, M, k=1,2$ means the up- and down-chirps, and the integration domains are shifted with $T_{c} / 2$ due practical reasons. In noiseless case when $k=1$ (up-chirp) the baseband equivalent on the receiver's input in the $i$-th slot is:

$$
r_{e k v}(t)=A \exp \left(2 j \pi \frac{\Delta f}{2 T_{c}}\left(t-\tau_{i}\right)^{2}\right)
$$

thus, after substituting in Eq. 8, one can get:

$$
\begin{aligned}
z_{i_{1}}= & \left|\int_{-\frac{T_{c}}{2}+\tau_{i}}^{\frac{T_{c}}{2}+\tau_{i}} r_{e k v}(t) g_{c i_{1}}^{*}(t) d t\right|^{2}= \\
& A^{4} \mid \int_{-\frac{T_{c}}{2}+\tau_{i}}^{\frac{T_{c}}{2}+\tau_{i}} \exp \left(2 j \pi \frac{\Delta f}{2 T_{c}}\left(t-\tau_{i}\right)^{2}\right) \\
& \left.\exp \left(-2 j \pi \frac{\Delta f}{2 T_{c}}\left(t-\tau_{i}\right)^{2}\right) d t\right|^{2}= \\
& A^{4} T_{c}^{2}=4 E_{s}^{2},
\end{aligned}
$$

where $E_{s}$ is the symbol energy expressed as:

$$
E_{s}=\frac{1}{2} A^{2} \int_{-\frac{T_{c}}{2}+\tau_{i}}^{\frac{T_{c}}{2}+\tau_{i}}\left|g_{c i_{k}}(t)\right|^{2} \text { for } k=1,2
$$


Furthermore,

$$
\begin{aligned}
z_{i_{2}}=\left|\int_{-\frac{T_{c}}{2}+\tau_{i}}^{\frac{T_{c}}{2}+\tau_{i}} r_{e k v}(t) g_{c i_{2}}^{*}(t) d t\right|^{2}= \\
A^{4} \mid \int_{-\frac{T_{c}}{2}+\tau_{i}}^{\frac{T_{c}}{2}+\tau_{i}} \exp \left(2 j \pi \frac{\Delta f}{2 T_{c}}\left(t-\tau_{i}\right)^{2}\right) \\
\left.\quad \exp \left(2 j \pi \frac{\Delta f}{2 T_{c}}\left(t-\tau_{i}\right)^{2}\right) d t\right|^{2} \approx 0,
\end{aligned}
$$

and for every $i^{*}$ (if $i \neq i^{*}, k=1,2$ )

$$
\begin{aligned}
z_{i_{k}^{*}}= & \left|\int_{-\frac{T_{c}}{2}+\tau_{i}^{*}}^{\frac{T_{c}}{2}+\tau_{i}^{*}} r_{e k v}(t) g_{c i_{k}^{*}}^{*}(t) d t\right|^{2}= \\
& A^{4} \mid \int_{-\frac{T_{c}}{2}+\tau_{i}^{*}}^{\frac{T_{c}}{2}+\tau_{i}^{*}} \exp \left(2 j \pi \frac{\Delta f}{2 T_{c}}\left(t-\tau_{i}\right)^{2}\right) \\
& \left.\exp \left( \pm 2 j \pi \frac{\Delta f}{2 T_{c}}\left(t-\tau_{i}^{*}\right)^{2}\right) d t\right|^{2} \approx 0 .
\end{aligned}
$$

This means that the output of the other detectors, which do not receive any useful signal, is approximately zero.

The following example justifies this hypothesis. Assume that $T_{c}=1, \Delta f=200, A=1, z_{i_{1}}=4 E_{s}^{2}=1$, then

$$
\begin{aligned}
z_{i_{2}} & =A^{4}\left|\int_{-\frac{T_{c}}{2}+\tau_{i}}^{\frac{T_{c}}{2}+\tau_{i}} \exp \left(4 j \pi \frac{\Delta f}{2 T_{c}}\left(t-\tau_{i}\right)^{2}\right) d t\right|^{2} \\
& =2.39 \cdot 10^{-3} \ll 1
\end{aligned}
$$

and

$$
z_{i_{k}^{*}}=\left|\int_{-\frac{T_{c}}{2}+\tau_{i}^{*}}^{\frac{T_{c}}{2}+\tau_{i}^{*}} r_{e k v}(t) g_{c i_{k}^{*}}^{*}(t) d t\right|^{2} \approx 5 \cdot 10^{-3} \ll 1,
$$

when the difference of delays is above $\left|\tau_{i}-\tau_{i}^{*}\right| \geq 0.03 \cdot T_{c}$.

Hence, the less the correlation of elementary signals are, the more the two messages can be distinguished from each other, i. e. the signals are almost orthogonal. By definition full orthogonality is only achieved, when the correlation equals exactly to zero. Nevertheless, in this case the correlation approximates it very closely.

Now, the behavior of the wireless channel will be taken into account, namely the disturbing effect of the random noise. The decision determining $z_{i_{k}}$ variables in the non-coherent nonbinary chirp receiver can be described by Eq. 8 as presented earlier. Suppose that the baseband equivalent of the input of the receiver is expressed as follows:

$$
e_{e k v}(t)=A e^{j \theta} g_{c i_{k}}(t)+n_{I}(t)+j n_{Q}(t),
$$

where $k=1,2, \theta$ is the unknown phase-shift of the radio channel, $n_{I}(t)$ and $n_{Q}(t)$ are the real and imaginary components of the Additive White Gaussian Noise (AWGN). $n_{I}(t)$ and $n_{Q}(t)$ are independent normal distributed stochastic processes, each has the power density $N_{0}$ and the autocorrelation function $N_{0} \delta(t)$. The attenuation of the channel is neglected, because it is already included in the Signal-to-Noise Ratio (SNR) during the calculation of the effective signal's power. $z_{i_{1}}$ and $z_{i_{2}}$ can be described in this case by the following expressions:

$$
\begin{aligned}
z_{i_{1}}= & \left|\int_{-\frac{T_{c}}{2}+\tau_{i}}^{\frac{T_{c}}{2}+\tau_{i}} r_{e k v}(t) g_{c i_{1}}^{*}(t) d t\right|^{2}= \\
& \left|\int_{-\frac{T_{c}}{2}+\tau_{i}}^{\frac{T_{c}}{2}+\tau_{i}}\left[A e^{j} \theta g_{c i_{1}}(t)+n_{I i}(t)+j n_{Q i}(t)\right] g_{c i_{1}}^{*}(t) d t\right|^{2}= \\
& \left.\left|\int_{-\frac{T_{c}}{2}+\tau_{i}}^{\frac{T_{c}}{2}+\tau_{i}} A^{2} e^{j} \theta\right| g_{c i_{1}}(t)\right|^{2} d t+ \\
& \int_{\frac{T_{c}}{2}+\tau_{i}}^{\frac{T_{c}}{2}+\tau_{i}} n_{I i}(t) g_{c i_{1}}^{*}(t) d t+\left.j \int^{\frac{T_{c}}{2}+\tau_{i}} n_{Q i}(t) g_{c i_{1}}^{*}(t) d t\right|^{2}= \\
& \left|2 e^{j \theta} E_{s}+n_{I i_{1}}+j n_{Q i_{1}}\right|^{2},
\end{aligned}
$$

and

$$
\begin{aligned}
z_{i_{2}}= & \left|\int_{-\frac{T_{c}}{2}+\tau_{i}}^{\frac{T_{c}}{2}+\tau_{i}} r_{e k v}(t) g_{c i_{2}}^{*}(t) d t\right|^{2}= \\
& \left|\int_{-\frac{T_{c}}{2}+\tau_{i}}^{\frac{T_{c}}{2}+\tau_{i}}\left[A e^{j} \theta g_{c i_{2}}(t)+n_{I i}(t)+j n_{Q i}(t)\right] g_{c i_{2}}^{*}(t) d t\right|^{2}= \\
& \mid \int_{-\frac{T_{c}}{2}+\tau_{i}}^{\frac{T_{c}}{2}+\tau_{i}} A^{2} e^{j} \theta g_{c i_{1}}(t) g_{c i_{2}}^{*}(t) d t+ \\
& \int_{-\frac{T_{c}}{2}+\tau_{i}}^{\frac{T_{c}}{2}+\tau_{i}} n_{I i}(t) g_{c i_{2}}^{*}(t) d t+\left.j \int_{-\frac{T_{c}}{2}+\tau_{i}}^{\frac{T_{c}}{2}+\tau_{i}} n_{Q i}(t) g_{c i_{2}}^{*}(t) d t\right|^{2} \approx \\
& \left|n_{I i_{2}}+j n_{Q i_{2}}\right|^{2},
\end{aligned}
$$

where $E_{s}$ is symbol energy as earlier, $n_{I i_{1}}, n_{I i_{2}}, n_{Q i_{1}}$ and $n_{Q i_{2}}$ are almost independent Gaussian distributed probability variables with zero expected value and with $2 E_{s} N_{0}$ variation. The independency of noise component can be only fulfilled, if the $g_{c i_{1}}(t)$ and $g_{c i_{2}}(t)$ signal pair would be exactly orthogonal. Nevertheless, assuming independency is applicable in practical cases due to the small absolute value of the correlation.

Note that the peak related to $\tau=0$ position follows Rice distribution in AWGN channel, which is illustrated in Fig. 8. The Rice distribution is given by the following formula:

$$
\begin{gathered}
f_{Y}(y)=\exp \left(-\left(y+\gamma_{0}\right)\right) I_{0}\left(2 \sqrt{y \gamma_{0}}\right), \\
\gamma_{0}=\frac{E_{s}}{N_{0}}, \quad Y=\frac{z_{i_{1}}(0)}{E_{s} N_{0}},
\end{gathered}
$$

where $I_{0}(\bullet)$ is the zeroth-order modified Bessel function and $\gamma_{0}$ is the SNR.

It is also a consequence of the above described formulae that on the normalized output of every detector, which did 


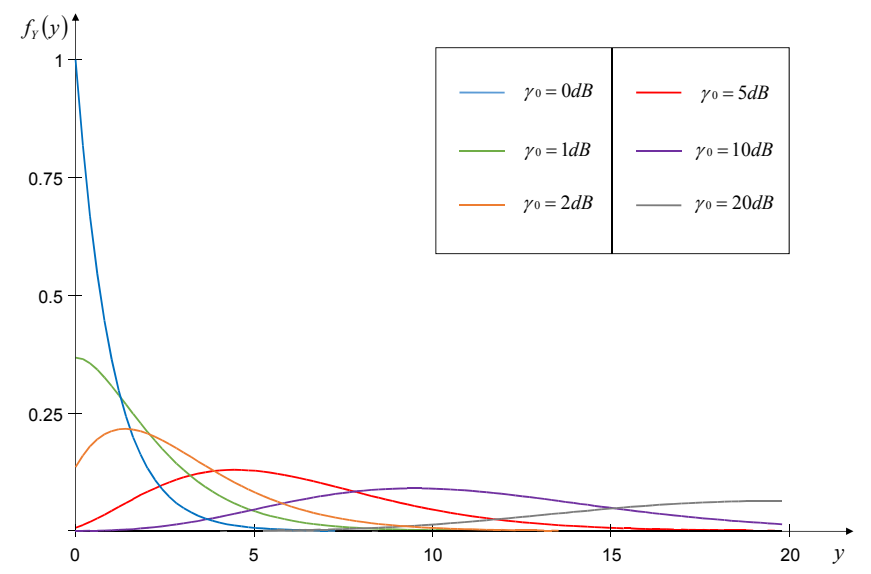

Figure 8. PDF of pulse's peak value observable on the output of the receiver supposing different SNR values

not receive any useful signal, i.e. $z_{i_{2}}(0)$ and $z_{l_{k}}(0)$ for $l=$ $1, \ldots, M$ and $k=1,2$, the probability density function (PDF) follows Rayleigh distribution identically and independently:

$$
f_{Y}(y)=\exp (-y) \text {. }
$$

\section{Performance Analysis And Numerical Results}

This section introduces the performance analysis of the proposed CSS-PPM-MA and CLPC techniques. The investigations focus on instantaneous and average Symbol-to-ErrorRatio (SER). In addition, theoretical results are presented regarding the efficiency of the measurement based feedback power control mechanism.

\section{A. Performance and evaluation of downlink}

To analyse the performance of CSS-PPM-MA method properly, the joint PDF of $z_{i_{k}}, i=1,2, \ldots, M, k=1,2$ probability variables has to be determined. Furthermore, the probability of the wrong decision has to be investigated to calculate the exact symbol-error ratio. This event formally happens if one from $z_{i_{2}}, z_{i_{k}^{*}}, i \neq i_{*}$ is higher than $z_{i_{1}}$, when $g_{c i_{1}}(t)$ (i. e. the $i$ th delayed, up-chirp symbol) was sent over the radio channel. Due to the non-coherent receiver structure of PP-CSS-MA, the following general expression for non-coherent reception in AWGN channel can be applied from [28]:

$$
\begin{aligned}
P_{S E R_{D L}}= & \sum_{m=1}^{2 M-1}(-1)^{m+1}\left(\begin{array}{c}
2 M-1 \\
m
\end{array}\right) \frac{1}{m+1} \\
& \exp \left(-\frac{E_{s}}{N_{0}} \frac{m}{m+1}\right),
\end{aligned}
$$

where $P_{S E R_{D L}}$ is the symbol-error rate for downlink. The SER for different data rates (i.e. different $M$ parameters) are shown on Fig. 9. The results for traditional non-coherent communication equals to the $M=1$ case. It is clearly visible and also unequivocal that increasing binary data rate carried by one symbol requires higher SNR to achieve the same SER. However, the difference in SNR between $M=1$ and $M=4$ is at most about $5 d B$ around $0 d B$ and getting less in higher

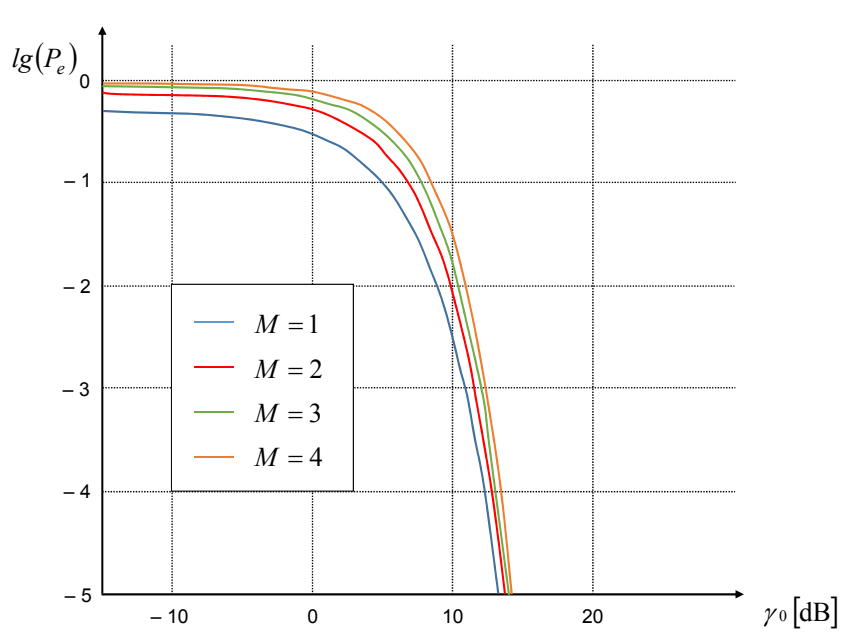

Figure 9. SER of the traditional non-coherent systems $(M=1)$ and the PP-CSS-MA technique $(M \geq 2)$

SNR domains. The calculation and the figure basically tell the price that have to be paid for the simultaneous transmission to more than one mobile terminal. This means that compared to the traditional CSS, to achieve the same SNR level and the same communication distance, the transmission power have to be slightly increased (by $2-5 d B$ ). The other option is to decrease the communication distance, while the same SNR and transmission power levels can be maintained. Reduction of the distance depends on the actual propagation attributes of the communication environment.

Beside the instantaneous SER, the average SER can be determined for slow, frequency non-selective Rayleigh fading channel by the following formula based on [28]:

$$
\begin{aligned}
\bar{P}_{S E R_{D L}}= & \sum_{m=1}^{2 M-1}(-1)^{m+1}\left(\begin{array}{c}
2 M-1 \\
m
\end{array}\right) \\
& \frac{1}{m+1} \frac{1}{1+\frac{m}{1+m} \frac{\bar{E}_{s}}{N_{0}}},
\end{aligned}
$$

where $\bar{E}_{s}$ is the average symbol energy of the signal and $\bar{P}_{S E R_{D L}}$ is the average SER for downlink. The average SER of the proposed CSS-PPM-MA technique in Rayleigh fading channel using different data rates is depicted in Fig. 10. Like on the previous figure, $M=1$ means the traditional CSS based communication. Similar relations can be found in this case, however, the deviation between the data rates become constants in the higher SNR domains. The relevance of these results is to anticipate the performance of the CSS-PPM-MA based system in such environment, where is no dominant propagation along a line of sight between the transmitter and receiver.

\section{B. Performance and evaluation of uplink}

For the exact error analysis, the joint PDF of $z_{i_{k}}, i=$ $1,2, \ldots, M, k=1,2$ probability variables and the probability of the wrong decision have to be determined again. The same approach is used as in case of the downlink analysis. 


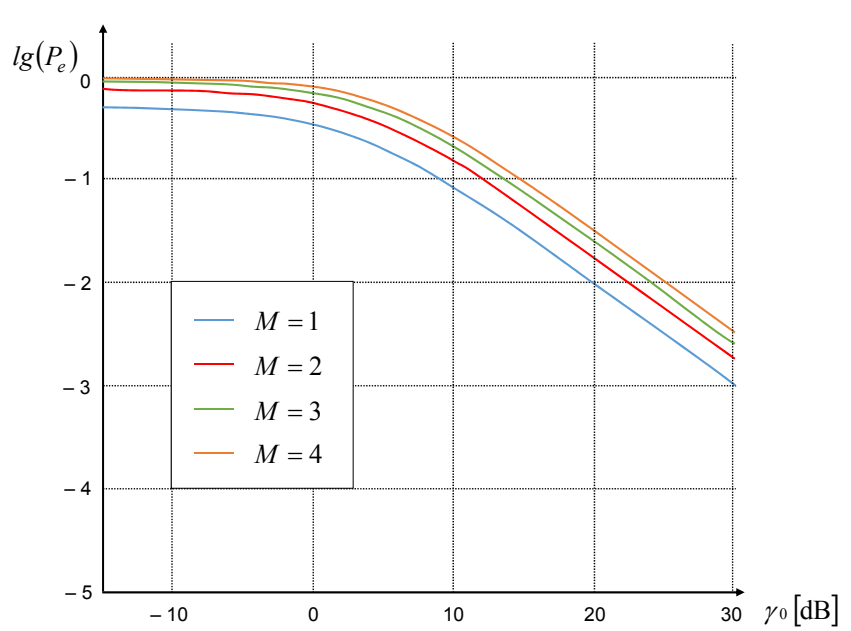

Figure 10. Average SER of the traditional non-coherent systems $(M=1)$ and the PP-CSS-MA technique $(M \geq 2)$ in Rayleigh fading channel

Thus, the solution of the instantaneous symbol-error rate is the following:

$$
P_{S E R_{U L}}=\frac{1}{2} \exp \left(-\frac{E_{s}}{2 N_{0}}\right)
$$

where $P_{S E R_{U L}}$ is the symbol-error rate for the uplink communication. Note, that this equation equals to Eq. 22 when $M=1$.

Similarly, the average SER in slow, frequency non-selective Rayleigh fading channel is expressed as:

$$
\bar{P}_{S E R_{U L}}=\frac{1}{2+\frac{\bar{E}_{s}}{N_{0}}}
$$

where $\bar{P}_{S E R_{U L}}$ is the average SER of uplink. Both curves are visible in Fig. 9 and Fig. 10 in case of $M=1$.

For extensive investigations, the influence of the quasiorthogonality on the demodulator has to be analysed as well. In other words the operation of the receiver has to be investigated when the correlation of the two elementary signals $g_{c_{1}}(t)$ and $g_{c_{2}}(t)$ is $\rho$. The solution of this problem is known from [28] for the channel without fading:

$$
\begin{aligned}
P_{b}= & {\left[\sum_{k=0}^{\infty}\left(\frac{1-\sqrt{1-\rho^{2}}}{1+\sqrt{1-\rho^{2}}}\right)^{\frac{k}{2}} \times I_{k}\left(|\rho| \times \frac{E_{b}}{2 N_{0}}\right)\right.} \\
& \left.-\frac{1}{2} I_{0}\left(|\rho| \times \frac{E_{b}}{2 N_{0}}\right)\right] \times \exp \left(-\frac{E_{b}}{2 N_{0}}\right),
\end{aligned}
$$

where $P_{b}$ is the bit-error rate (BER) and $I_{k}(\bullet)$ is the k-th order modified Bessel function. The results are depicted in Fig. 11 for different correlation parameters. Based on the figure the effect of correlation between the elementary signals can be neglected in the proposed system, if $|\tau| \geq 0.015 T_{c}$.

As mentioned, the original measurement based feedback power control mechanism from [27] is slightly changed and aligned to the nature of PP-CSS based communication system, but still, the results are directly applicable. Therefore, the details of the calculations are not presented in this paper.

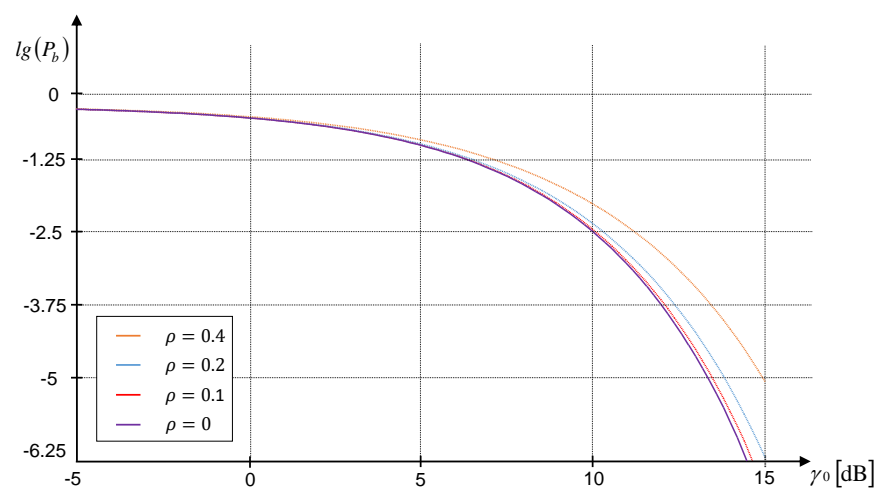

Figure 11. BER of non-coherent binary receiver depending on the SNR for different correlation parameters $(\rho)$

Only the results focusing on Rayleigh fading channel are highlighted, however, [27] contains results related to other major fading types, like Nakagami-m and Rician fading.

The bit-error rate of the proposed measurement based feedback power control mechanism assuming Rayleigh fading channel is given as follow:

$$
P_{b_{C L P C}}\left(\gamma_{s}\right)=\int_{0}^{\infty} \frac{1}{h(y)} \frac{\gamma_{0}}{1+\gamma_{0}} \exp \left(-\frac{\gamma_{0}}{1+\gamma_{0}} y\right) d y
$$

where $P_{b_{C L P C}}\left(\gamma_{s}\right)$ is the average BER, $\gamma_{s}$ is the SNR of the channel, $\gamma_{0}$ is the SNR of the pilot signal, $y$ describes the stochastic process of the fading parameter estimation:

$$
Y=\left|z+\frac{\overline{\mathbf{n}}}{\sqrt{E_{0}}}\right|^{2},
$$

and the $h(x)$ function introduces the threshold value related to the maximum transmission power of the mobile terminals:

$$
h(x)=\left\{\begin{array}{c}
c \text { if }|x| \leq c \\
|x|^{2} \text { if }|x|>c
\end{array}\right.
$$

Basically, Eq. 27 represents the behaviour of the uplink communication taking into account the limitations of the mobile terminals regarding their transmission power and the estimation error due to the imperfect reception of the pilot (i. e. the frame synchronizing down-chirp) signal. The numerical results are depicted in Fig. 12 and Fig. 13. The former figure illustrates the effect of changing the transmission power related threshold value in Rayleigh fading channel. The latter one shows the bit-error rate depending on different level of the pilot signal's SNR. On both figures the theoretical BER of traditional, unequalized communication (that can be a binary CSS or a binary PPM based system) is depicted by blue color. Compared to that systems the proposed CLPC mechanism is able to significantly increase the performance of the uplink communication. In some cases it can even exceed the performance of diversity usage as well. It is visible that the limits of mobile terminals and the quality of pilot signal, i. e. how effectively the MT can eliminate the distortions of the radio channel, have strong impact on the communication. 


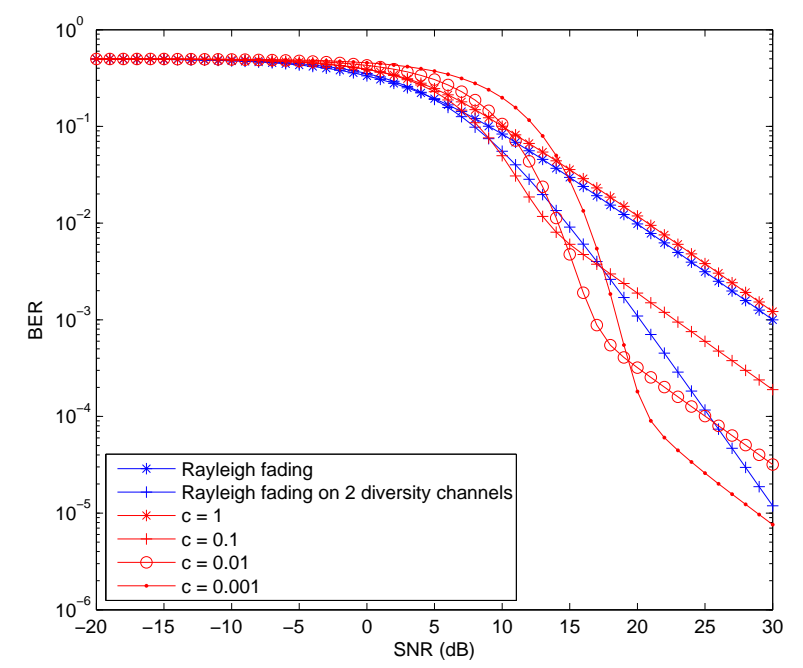

Figure 12. Numerical results of average BER of Rayleigh fading channel against the average SNR, when the SNR of the pilot signal is $\gamma_{0}=30 \mathrm{~dB}$ and the power limiting factors are $c=1-0.001$.

\section{DISCUSSION}

This section summarizes the pros and cons of the proposed system, and overviews some important practical aspects.

The realization of the described concept does not require complex signal processing compared to the traditional CSS based transmitter and receiver, while changing the chirp signal attributes proposed by other multiple access schemes might result in difficult transmitter and/or receiver structure. For effective communication effective management of the power amplifier is necessary especially in LPWAN systems. By investigating this issue, the PP-CSS-MA technique just slightly increases the peak-to-average power ratio (PAPR) at the base station side compared to the original CSS based communication. Nevertheless, the growth of PAPR is much smaller than in case of fully overlapping signals. Similarly, the transmission power limitation of the mobile terminals is introduced by the $c$ parameter during the analysis of the CLPC mechanism. This parameter describes the natural behaviour that a transmitter is not able to compensate completely in every case the distortion of the radio channel. For example when very large or infinite amplitude compensation is required, then an MT can raise its transmission power only to its limit. These practical aspects are usually not considered by the related works.

Assuming the PP-CSS-MA variant A the down-chirp signal has two purposes in the system. On the one hand, it provides the frame synchronization for the mobile terminals. On the other hand, it functions as pilot signal for the CLPC mechanism. Therefore it is a sophisticated solution to connect these two concepts in the proposed communication system.

The advantages of the proposed downlink communication are the following: $I$. the base station is able to communicate with more than one mobile terminals simultaneously; II. the transmitter sends basically only one elementary signal at a time, thus the transmission power only slightly grows as $M$ increases; III. compared to the traditional CSS, the CSS-PPM-

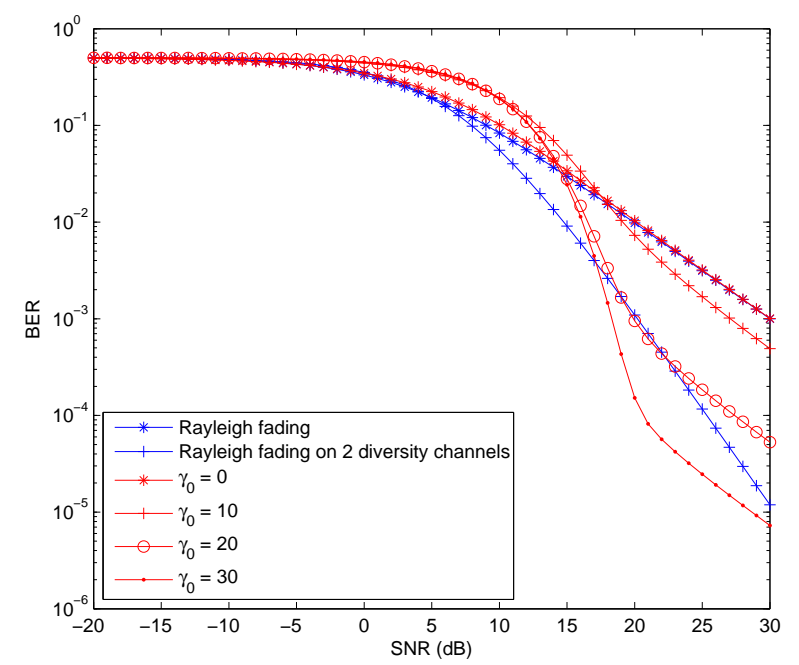

Figure 13. Numerical results of average BER of Rayleigh fading channel against the average SNR, when the SNRs of the pilot signal are $\gamma_{0}=0-30$ $\mathrm{dB}$ and the power limiting factor is $c=0.001$.

MA technique does not require to significantly increase the transmission power to maintain the same coverage.

Contrarily, the drawbacks are as follows: $I$. the elementary signals with different delays transmitted in neighbouring slots can overlap as mentioned (see Fig. 2), due to this behavior the PAPR of the resultant signal increases, i.e. the instantaneous power of the transmitter grows; II. as visible from the receiver structures (Fig. 3 and Fig. 4) lots of correlators $\left(2^{M}\right.$ in case of CSS-PPM-MA variant A and $2 M$ in case of CSS-PPMMA variant B) are necessary in the receiver that significantly improves its complexity. However, applying matched filters instead of the correlators can simplify the structure. In case of variant $\mathrm{A}$ one filter is matched to the down-chirp and one to the up-chirps. The latter one is able to detect all the pulseposition based signals, i. e. all the time-shifted up-chirps, using a sliding time-window. Similarly, in case of variant B two matched filters are capable of handling the input signals.

The benefits of the proposed uplink communication are the following: $I$. every mobile terminal is able to communicate with the base station simultaneously; II. the transmitter of every MT sends only one elementary signal at a time, this allows the transmission power be the same compared to the traditional chirp spread spectrum, i. e. the uplink communication range is not affected; III. the system is barely sensitive to the fluctuations of time delay, i.e. to the synchronization errors (see Fig. 6 and Fig. 7). The base station can provide the time synchronization.

The disadvantage of the CSS-PPM based uplink communication is that transmission power control is required in the mobile terminals to tackle the near-far problem, however, this issue is handled by the proposed CLPC mechanism.

An additional remark that in case of PP-CSS-MA variant B the downlink and the uplink channels are not symmetrical, since during one symbol time $1+\log _{2} M$ bit information can be transmitted to the mobile terminals, while they can send $M$ 
data bits to the base station. Contrarily, the throughput of both the downlink and uplink channels are $M$ regarding variant A.

\section{SUMMARY}

In the present paper, a novel transmission method, the pulse position based chirp spread spectrum was introduced. For downlink communication two multiple access variants were presented based on this technique, while for uplink, beside the PP-CSS method, the measurement based feedback power control was proposed for use to handle the amplitude control of overlapping quasi-orthogonal chirp signals at the base station. In addition, analytical investigations were provided for the instantaneous and average symbol-error rates as well as for average bit-error rates assuming Rayleigh fading channel. The results show that efficient communication can be established between the base station and multiple mobile terminals using the proposed techniques.

\section{REFERENCES}

[1] Cisco, Cisco Visual Networking Index: Global Mobile Data Traffic Forecast Update, 2016-2021 White Paper, March 2017, https://tinyurl.com/zjpd2go

[2] Ericsson, Ericsson Mobility Report, November 2016 , https://tinyurl.com/jeet6pg

[3] J. G. Andrews et al., "What Will 5g Be?," IEEE Journal on Selected Areas in Communications, vol. 32, no. 6, pp. 1065-1082, June 2014 DOI: $10.1109 /$ JSAC.2014.2328098

[4] J. Rodriguez, Fundamentals of $5 G$ Mobile Networks, 1st edition, John Wiley \& Sons, 2015. ISBN: 978-1-118-86752-5

[5] A. Osseiran et al., 5G Mobile and Wireless Communications Technology, Cambridge University Press, 2016. ISBN: 978-1107130098

[6] E. Karapistoli et al., "An overview of the IEEE 802.15.4a standard," IEEE Communications Magazine, vol. 48, no. 1, pp. 47-53, January 2010. DOI: $10.1109 /$ MCOM.2010.5394030

[7] IEEE 802.15.4-2011 Standard, http://www.decawave.com/technology/ieee802154a-standard

[8] LoRa Alliance, LoraWAN Specification, V1.0.2, July 2016.

[9] Mobile Experts, White Paper for LoRa Alliance, April 2016.

[10] Merrill Ivan Skolnik, Introduction to Radar Systems, Mcgraw-Hill College, 2nd edition, 1980. ISBN: 978-0070579095

[11] C. E. Cook, "Linear FM Signal Formats for Beacon and Communication Systems," in IEEE Transactions on Aerospace and Electronic Systems, vol. AES-10, no. 4, pp. 471-478, July 1974. DOI: 10.1109/TAES.1974.307800

[12] S. E. El-Khamy, S. E. Shaaban and E. A. Tabet, "Efficient multipleaccess communications using multi-user chirp modulation signals," Spread Spectrum Techniques and Applications Proceedings, 1996., IEEE 4th International Symposium on, Mainz, 1996, pp. 1209-1213 vol.3. DOI: $10.1109 /$ ISSSTA.1996.563498

[13] S. Hengstler et al., "A novel chirp modulation spread spectrum technique for multiple access," IEEE Seventh International Symposium on Spread Spectrum Techniques and Applications, 2002, pp. 73-77 vol.1. DOI: 10.1109/ISSSTA.2002.1049289

[14] Y. Ju and B. Barkat, "A new efficient chirp modulation technique for multi-user access communications systems," 2004 IEEE International Conference on Acoustics, Speech, and Signal Processing, 2004, pp. iv937-40 vol.4. DOI: 10.1109/ICASSP.2004.1326982

[15] B. Reynders and S. Pollin, "Chirp spread spectrum as a modulation technique for long range communication," 2016 Symposium on Communications and Vehicular Technologies (SCVT), Mons, 2016, pp. 1-5. DOI: 10.1109/SCVT.2016.7797659

[16] M. A. B. Othman et al., "Exploiting Quasi-Orthogonal Chirp Signals in Multi-User Access Communication Systems," 2011.
[17] S. E. El-Khamy, S. E. Shaaban and E. A. Thabet, "Frequency hoppedchirp modulation (FH-CM) for multi-user signaling in multipath dispersive media," IEEE Antennas and Propagation Society International Symposium. 1999 Digest. Held in conjunction with: USNC/URSI National Radio Science Meeting (Cat. No.99CH37010), Orlando, FL, USA, 1999. pp. 396-399 vol.1. DOI: 10.1109/APS.1999.789162

[18] "AN1200.22 LoRa ${ }^{\mathrm{TM}}$ Modulation Basics," Rev. 2, Semtech, May 2015.

[19] D. Bankov, E. Khorov and A. Lyakhov, "On the Limits of LoRaWAN Channel Access," 2016 International Conference on Engineering and Telecommunication (EnT), Moscow, 2016, pp. 10-14. DOI: 10.1109/EnT.2016.011

[20] G. L. Stuber, Principles of Mobile Communication, 2nd ed. Norwell, MA: Kluwer, 2001. ISBN: 978-0792379980

[21] Erik Dahlman, Stefan Parkvall, Johan Skold, 4G: LTE/LTE-Advanced for Mobile Broadband, 2nd edition, Academic Press, October 2013, ISBN: 9780124199859.

[22] G. Zhao, G. Y. Li, and C. Yang, "Proactive detection of spectrum opportunities in primary systems with power control," IEEE Trans. Wireless Commun., vol. 8, no. 9, pp. 4815-4823, September 2009. DOI: 10.1109/TWC.2009.081585

[23] F. E. Lapiccirella, S. Huang, X. Liu, and Z. Ding, "Feedback-based access and power control for distributed multiuser cognitive networks," in Proc. IEEE ITA, San Diego, CA, Feb. 2009, pp. 85-89. DOI: 10.1109/ITA.2009.5044927

[24] L. Mendo and J. M. Hernando, "Improved Algorithm for Computation of Transmission Powers in DS-CDMA Cellular Networks with ClosedLoop Power Control," 2008 IEEE 68th Vehicular Technology Conference, Calgary, BC, 2008, pp. 1-5. DOI: 10.1109/VETECF.2008.388

[25] M. Dosaranian-Moghadam, H. Bakhshi, G. Dadashzadeh and M. Godarzvand-Chegini, "Joint closed-loop power control and constrained LMS algorithm for DS-CDMA receiver in multipath fading channels," 2010 Global Mobile Congress, Shanghai, 2010, pp. 1-8. DOI: 10.1109/GMC.2010.5634558

[26] Á. Knapp and L. Pap, "Performance analysis of pulse position based chirp spread spectrum technique for multiple access," 2017 25th International Conference on Software, Telecommunications and Computer Networks (SoftCOM), Split, 2017, pp. 1-5. DOI: 10.23919/SOFTCOM.2017.8115574

[27] Á. Knapp and L. Pap, "General Performance Analysis of Binary Fading Channels with Measurement Based Feedback Channel Equalization," Infocommunications Journal, vol. 6, no. 1, pp. 1-9, March 2014.

[28] John Proakis and Masoud Salehi, Digital Communications, 5th edition, McGraw-Hill, November 2007, ISBN: 0072957166.

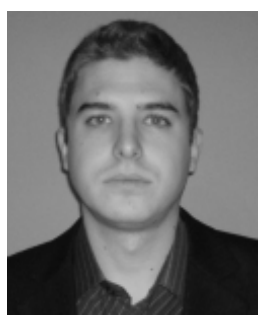

Ádám Knapp received his M. Sc. degree in software engineering in 2011 from the Budapest University of Technology and Economics, Hungary. At the present, he is PhD student at BUTE, Dept. of Networked Systems and Services. His main research topic includes communication theory, energy efficiency and QoS in NGN networks and network simulation of LTE and LTE-A.

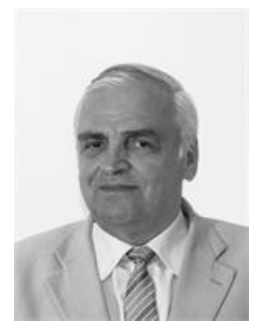

László Pap graduated from the Technical University of Budapest, Faculty of Electrical Engineering, Branch of Telecommunications. He became Dr. Univ. and Ph.D. in 1980, and Doctor of Sciences in 1992. In 2001 and 2007 he has been elected as a Correspondent and Full Member of the Hungarian Academy of Sciences. His main fields of the research are the electronic systems, nonlinear circuits, synchronization systems, modulation and coding, spread spectrum systems, CDMA, multiuser detection and mobile communication systems. His main education activity has covered the fields of electronics, modern modulation and coding systems, communication theory, introduction to mobile communication. Professor Pap had been Head of the Dept. of Telecommunications, the Dean of the Faculty of Electrical Engineering at Budapest University of Technology and Economics, and Vice Rector of the University. 